\title{
Zwischen Programm und Populismus - die offene Zukunft der CSU
}

von Heinrich Oberreuter

\begin{abstract}
Die jüngere Entwicklung der CSU ist durch eine fundamentale Paradoxie gekennzeichnet: Einerseits wurde sie von den gleichen gesellschaftlichen Modernisierungsprozessen erfasst, die die übrigen Parteien bereits seit längerem vor neue Herausforderungen stellen. Andererseits ist sie deutlich stärker von einer Reihe spezifischer Kontextbedingungen abhängig, die ihre Sonderrolle im deutschen Parteiensystem untermauert. Der nachfolgende Beitrag befasst sich mit der Frage, ob der CSU zunehmend das zum Erhalt dieser Sonderrolle notwendige soziale Substrat abhanden kommt und welche Kompensationsstrategien erkennbar sind. Die schwierige Entwicklung der Partei seit dem Ende der „Ära Stoiber” wird im Kontext ihres internen Organisationsgefüges, ihrer bundes- und europapolitischen Wirkung und ihrer programmatischen Aufstellung erörtert. Dies bildet die Grundlage für eine zusammenfassende Zustandsbeschreibung und den Ausweis erkennbarer Handlungsoptionen.
\end{abstract}

The CSU's recent development is characterised by a fundamental paradox: on the one hand, the party continues to be affected by the same processes of social modernisation that have been challenging other political actors for some time. On the other hand, it is disproportionately more dependent on a series of specific contexts constituting the foundation of its special position in the German party system. This contribution examines the state of these foundations and stipulates that the erosion of the party's social substrate could endanger its long-standing "Sonderrolle". Subsequently, the CSU's difficult development since the end of the "Stoiber era" is discussed in the context of the party's internal organisation, its role in federal and European politics, and its programmatic line-up. This leads to a concluding description of the party's current state and its potential future development.

\section{Tradition und Situation: die Paradoxie der CSU}

Es steht wohl außer Zweifel, dass die CSU auch jenseits des deutschen Parteiensystems das „Musterbeispiel“ einer eigenständigen, keineswegs provinziellen und überdurchschnittlich erfolgreichen Regionalpartei darstellt. Dabei ist ihre derzeitige Lage durch eine nicht unproblematische Paradoxie gekennzeichnet: Einerseits wurde die Partei „normaler“ und in ihren Problemlagen den anderen (Volks-)Parteien vergleichbarer. Gesellschaftliche Modernisierungs- und Säkula- 
risierungsprozesse ${ }^{1}$ haben auch Bayern erfasst und Spezifika der vorherrschenden Lebensstile und Lebensgefühle nivelliert - sofern deren Prägekraft nicht ohnehin stets überinterpretiert wurde, man das Altbayerische gleichsam zum Prototyp des Bayerischen erhob. Traditionszonen und mentale, aus den verschiedenen Volksstämmen erwachsene Pluralitäten sind nie verschwunden, sondern wurden von der CSU lediglich ,staatsbayerisch“ überwölbt. ${ }^{2}$ Mentalitäten und Traditionen lassen sich allerdings nicht unbedingt staatlich fassen, zumal es zunehmend fraglich erscheint, inwiefern diese Kategorien überhaupt noch politisch orientierend oder strukturierend wirken. Wahrscheinlich war die (polemische) These, derzufolge die CSU selbst erst das schöne Bayern erfand, ${ }^{3}$ schon immer überzogen.

Zudem hat sich die Gesellschaft deutlich entpolitisiert, was die politische Wirksamkeit überkommener Folklore substantiell und langfristig kaum steigern dürfte. Dazu tragen auch Wanderungsbewegungen bei, mit etwa 3,3 Millionen bzw. 1,3 Millionen Zuzügen aus anderen Bundesländern und dem Ausland allein zwischen 2000 und 2009. ${ }^{4}$ Verbunden mit Abwanderungen in vergleichbarem Umfang und innerbayerischer Migration vom Land in die Metropolen zeugt dies von erheblicher Bewegung in der Gesellschaft. Auch der historische Wandel von der Agrar- über die Industrie- zur Hochtechnologiestruktur sollte nicht in Vergessenheit geraten. So verzeichnet denn auch eine aktuelle, Traditionen und Traditionszonen nachspürende „Bayernstudie“ nur mäßige politische Zufriedenheit und eine lediglich schwach ausgeprägte Assoziation von ,guter Politik“ mit „Bayern“. ${ }^{5}$ Die Studie analysiert zudem die gesellschaftliche Definition des Freistaats jenseits seiner politischen Identität und ergründet generations-, geschlechts-, bildungs- und einkommensspezifische Positionen hinsichtlich der Frage, wie wichtig es den Teilnehmern ist, „dass Bayern politisch und wirt-

1 Zwischen 1970 und 2009 sank der Anteil der Katholiken von 70,4 auf 53,8 Prozent, der von Protestanten von 25,2 auf 20,9 Prozent. Es stieg der Anteil der Bekenntnislosen von 3,5 auf 22,7 Prozent und der Sonstigen von 0,9 auf 2,6 Prozent (vgl. Bund für Geistesfreiheit Bayern K.d.ö.R., http://www.bfg-bayern.de/tatsachen/kirchenaustrittbayern.htm [Stand: 24. Mai 2011], auf Grundlage der Angaben von Kirchen, des Statistischen Landesamtes Bayern sowie der Hochrechnung statistischer Einzeldaten).

2 So die treffende Analyse bei Mintzel, A.: Die CSU. Anatomie einer konservativen Partei, Opladen, 1975.

3 Riehl-Heyse, H.: CSU. Die Partei, die das schöne Bayern erfunden hat, München, 1979.

$4 \mathrm{Zu}$ den Daten siehe Bayerisches Landesamt für Statistik und Datenverarbeitung: Wanderungen über Landesgrenzen/Bundesgrenzen, <https://www.statistik.bayern.de/statistik/wanderungen> (Stand: 24. Mai 2011).

5 Projektgruppe: Fakten aus der Bayernstudie, in: Gruber, T. (Hg.): Ansichtssache Bayern. Annäherungen an eine Heimat, München, 12-30, insb. 21-24. 
schaftlich eine führende Rolle unter den Bundesländern spielt““. ${ }^{6}$ All das spricht für Situations-, nicht für Traditionsorientierung. Bayerns Gesellschaft hat sich nicht nur modernisiert - keineswegs ohne vielfaches Zutun der CSU -, sondern auch entpolitisiert. Insofern gehen Bayerns Uhren heute nur noch in geringem Maße ,anders“ als im Rest der Republik, um einen alten Streit aufzugreifen. ${ }^{7}$ Die CSU spürt die benannte Entwicklung vor allem an den Wahlurnen - bereits seit Längerem schleichend, seit 2008 eher dramatisch.

Andererseits zählt die von der Bevölkerung mit distanzierter Gelassenheit wahrgenommene (im Alltag aber durchaus geschätzte) Spitzenposition Bayerns im föderalen Wettbewerb nicht nur zum Kern des Selbstverständnisses der CSU (vgl. etwa die beliebte Metapher der „Champions League“). Vielmehr wurde sie auch historisch-pragmatisch zur substantiellen Voraussetzung der von der CSU seit langem beanspruchten und geprägten Sonderrolle als regional verankerter Partei mit bundespolitischem Anspruch. Erfolg in dieser Sonderrolle begründet die - derzeit geschwächte - hegemoniale Position in Bayern und den Einfluss im Bund. Es bleibt die Frage, ob dieser Sonderrolle zunehmend das gesellschaftliche Substrat abhanden kommt.

Jedenfalls muss sie neu definiert werden, wenn ihre Begründung immer mehr auf organisatorischen sowie funktionalen Argumenten beruht und zugleich die ehemals feste gesellschaftliche Verankerung aufzubrechen droht. Denn dann schwindet sowohl die benannte überkommen-diffuse Zustimmung als auch die häufig geduldete und weithin unterstellte Vorstellung einer Identität von Freistaat und Partei. Zustimmung erwächst heute eher aus einem situativen Leistungs- und Nutzenkalkül. Damit verliert die These, die Partei könne ihre Hegemonie in Bayern nur selbst gefährden, ${ }^{8}$ ihre apodiktische Kraft. Selbstgefährdung und Herausforderungen durch partiell auch selbstverantwortete Modernisierungsprozesse in den Bereichen Bildungssystem und Wirtschaftsentwicklung überlagern sich inzwischen. Die ehemals hegemoniale CSU sieht sich zwar weiterhin in einer dominanten, gleichwohl aber geschwächten Position, die immer

6 Ebd., 24.

7 Falter, J.W.: Bayerns Uhren gehen wirklich anders. Politische Verhaltens- und Einstellungsunterschiede zwischen Bayern und dem Rest der Republik, in: Zeitschrift für Parlamentsfragen, 13/4 (1982), 504521; Mintzel, A.: Gehen Bayerns Uhren wirklich anders?, in: Zeitschrift für Parlamentsfragen 18/1 (1987), 77-93; Falter, J.W.: Wie gehen sie denn nun, die bayerischen Uhren?, in: Zeitschrift für Parlamentsfragen, $19 / 1$ (1988), 113-114.

8 Mintzel, A.: Die CSU-Hegemonie in Bayern. Strategie und Erfolg. Gewinner und Verlierer, Passau, 1998, insb. 257-259. 
mehr auf ökonomisch-situativen Kompetenzzuschreibungen („Laptop“) als auf Spezifika der bayerischen Kultur („Lederhose“) beruht.

Diese Entwicklungen stürzten die Partei in den Wahljahren 2008 und 2009 in eine tiefe, verunsichernde Erfolgskrise: -5,5 Prozentpunkte bei den Kommunalwahlen im Frühjahr 2008, deren Menetekel keineswegs erkannt wurde, $-17,3$ bei den Landtagswahlen im Herbst, $-6,7$ bei den Bundestagswahlen 2009. Diese „Erdrutsche“ allein auf hausgemachte Oberflächenfaktoren - Stoibers Selbstdestruktion und sein Sturz, ${ }^{9}$ die unglücklich agierende und auf innerparteiliche Widerstände stoßende Doppelspitze Beckstein-Huber, kommunikative Defizite zurückzuführen, griffe zu kurz. Anscheinend gab die Stoiber-Beckstein-HuberKrise den Wählern endgültig den Anstoß, die benannten gesellschaftlichen Angleichungs- und Nivellierungsprozesse an den Wahlurnen zum Ausdruck zu bringen. Das diese Tendenzen bislang überlagernde Grundvertrauen wurde deutlich beschädigt.

Diese Erosion hatte sich, von der Partei unbemerkt, längst angekündigt. Selbst beim fulminanten Wahlsieg 2003 mit einer Zweidrittelmehrheit der Mandate im Landtag hatte die CSU über eine Viertelmillion an absoluten Stimmen verloren. Zwischen 1983 und 2009 sank die Zahl der Stimmen bei Bundestagswahlen von 4,1 (mit einem Zwischenhoch bei Stoibers Kanzlerkandidatur 2002: 4,3) auf 2,8 Millionen, bei den Landtagswahlen von 1986 bis 2008 von 6,3 auf 4,6 Millionen. ${ }^{10}$ Dafür ist nicht allein die sinkende Wahlbeteiligung verantwortlich.

Ein Blick auf das Jahr 2008 verdeutlicht, dass das „bürgerliche“ Lager in Bayern keineswegs geschrumpft ist. Es hat sich auf Kosten der CSU nur stark differenziert. Ihre absolute Mehrheit, zuvor in allen Gruppen der Bevölkerung erkennbar, vermochte sie allein bei den Rentnern zu verteidigen. Die Absatzbewegung erfasste Arbeiter, Angestellte und Beamte; mit -40 Prozentpunkten am deutlichsten aber die Landwirte. In den mittleren und jungen Altersgruppen liegen die Verluste durchweg um die 20, unter den Katholiken und Konfessionslosen bei etwa 17 und bei den bayerischen Protestanten um die 15 Prozentpunkte. Auch der Katholizismus bietet also keinen Halt mehr. Die Flächen wie auch Sozialstrukturen übergreifenden und (von den Landwirten abgesehen) wenig differenzierten Verluste verweisen auf generelle Vertrauenseinbußen. Ihr Ausmaß und ihre gleichmäßige soziostrukturelle Verteilung beschneiden die CSU auf ein

9 Oberreuter, H.: Stoibers Sturz. Ein Beispiel für die Selbstgefährdung politischer Macht, in: Zeitschrift für Parlamentsfragen, 39/1 (2008), 112-118.

10 Zur Ermittlung der Wahlergebnisse werden in Bayern die Erst- und Zweitstimmen zusammengezählt. 
noch immer sehr gehobenes Normalmaß. Gleichwohl wurde auch sie inzwischen von den Erosions-, Mobilisierungs- und Integrationsproblemen der anderen Volksparteien erfasst. Zielgerichtet auf wirtschaftliche Modernisierung hinarbeitend, zugleich sich aber auf vermeintlich unerschütterliche Traditionen stützend, verfehlte sie zunehmend die Lebenswirklichkeit bestimmter Bevölkerungsgruppen, darunter junge, berufstätige und gut ausgebildete Frauen. Die Zufriedenheit mit der Wirtschafts- und Sicherheitspolitik wurde überlagert von Kompetenzverlusten, vor allem bei den Themen Familie, Bildung und - trotz der Wirtschaftskompetenz - Arbeit. Die rein ökonomisch orientierte Modernisierungsrhetorik verpuffte und verfehlte den Wertewandel in der Wählerschaft. Indikatoren für eine Umkehr dieser Tendenzen zeigen sich nicht. Die zuletzt auch in Bayern sichtbare Volatilität der Wählerschaft spricht ohnehin gegen eine Rückkehr zum status quo ante.

\section{Metamorphosen: Ablösung vom sozialen Substrat}

Für die CSU ist dies eine Zäsur von historischem Ausmaß, das mit den üblichen tagesaktuellen parteipolitischen Interpretationen und Opportunismen nicht erfasst werden kann. Auch ist es bislang kaum ins Bewusstsein der politisch Handelnden getreten. Andererseits könnte sich die negative Wirkung in Grenzen halten, wenn die traditionelle Organisationsform Partei insgesamt in Erscheinung, Bedeutung, Wirkweise, Kommunikationstechnik, Kohäsion, sozialer Verwurzelung und Integration nicht nur ,unter Stress“ gerät, ${ }^{11}$ sondern Metamorphosen erfährt, die ihre ursprüngliche idée directrice (Maurice Hauriou) ${ }^{12}$ bis zur Unkenntlichkeit modifizieren. Im Ergebnis stünde die Partei als von Ideen und sozialstrukturellen Bindungen entkernte, gemäß der gesellschaftlichen Individualisierung stimmungs- und opportunitätszugewandte, mitgliederschwache, professionalisierte und personalisierte, d.h. auf populistische und wendungsbereite leader konzentrierte, sowie schließlich ggf. kontinuitätsvergessene, dafür aber machtorientierte Thematisierungs- und Mobilisierungsagentur.

Anzeichen einer derartigen Metamorphose werden seit Längerem diskutiert, ${ }^{13}$ ohne schon zu einem überzeugenden Modell aggregiert worden zu sein. Die

11 Plasser, F.: Parteien unter Stress. Zur Dynamik der Parteiensysteme in Österreich, der Bundesrepublik Deutschland und den Vereinigten Staaten, Wien u.a., 1987.

12 Hauriou, M.: Die Theorie der Institution und zwei andere Aufsätze, Berlin, 1965.

13 Katz R.S./Mair, P.: Changing Models of Party Organization and Party Democracy. The Emergence of the Cartel Party, in: Party Politics 1/1 (1995), 1-28; v. Beyme, K.: Parteien im Wandel. Von den Volksparteien zu den professionalisierten Wählerparteien, Wiesbaden, 2000; Oberreuter, H.: Image statt 
Parteien befinden sich mitten in diesem Prozess - auch die angeblich sozial so tief verwurzelte, traditionsorientierte CSU. Der Wurzelgrund hat sich gelockert. Wie andere Parteien auch verfügt sie über eine Führung, die die Wendungen des Zeitgeistes mit großer Aufmerksamkeit verfolgt. ${ }^{14}$ In Bayern ist dies auffälliger als andernorts, weil von dort bisher eher gegenteilige Signale ausgingen. Wenn das soziale Substrat durch modernisierende Nivellierungen abhanden gekommen ist, begründet dies für die CSU in der Tat Anpassungszwänge und Herausforderungen. Es ändert zwar wenig an ihren grundlegenden Aktionsbedingungen als Regionalpartei mit bundesweiten Ansprüchen, entzieht ihr jedoch quasi ,natürliche“, jedenfalls unterstützende und notwendige Voraussetzungen. Sehr zugespitzt: Die „bayerische“ Rolle fällt ihr nicht mehr kraft Identität zu, sondern bestenfalls durch Leistung, Bewährung und Sichtbarkeit als regional ,zuständiger" Akteur in der Bundes- und Europapolitik. Ihre organisatorische Selbständigkeit hebt sie von den Konkurrenten in Bayern ab, die nur als Landesverbände von Bundesparteien auftreten können, aber eben nicht als eigene Partei und Interessenvertretung auf nationaler und supranationaler Ebene. ${ }^{15}$ Diesen strukturellen Vorteil können auch die modernisierenden gesellschaftlichen Entwicklungen nicht gänzlich einebnen. Er wird aber erneut nicht durch Mentalitäten und Folklore konstituiert, sondern durch Interessen, und er kann nur über deren permanente Befriedigung nachhaltig wirken. So gewinnen die Erfolgskriterien performance, Tagesaktualität und Kurzzeitorientierung an Priorität.

\section{Sonderrolle als Existenzbedingung}

Bei allen Modifizierungen von Begründung und strategischem Inhalt der Sonderrolle bleibt sie und ihre wie auch immer erkennbare Ausgestaltung eine frühzei-

Inhalte? Möglichkeiten und Grenzen inszenierter Politik, in: Depenheuer, O. (Hg.): Öffentlichkeit und Vertraulichkeit. Theorie und Praxis der politischen Kommunikation, Wiesbaden, 2001, 145-157; Jun, U.: Der Wandel von Parteien in der Mediendemokratie. SPD und Labour Party im Vergleich, Frankfurt a.M./New York, 2004; Wiesendahl, E.: Parteien, Frankfurt a.M., 2006; Oberreuter, H./Kranenpohl, U.: Parteien, in: Gerlach, I./Jesse, E./Kneuer, M./Werz, N. (Hg.): Politikwissenschaft in Deutschland, Baden-Baden, 2010 [= Veröffentlichungen der Deutschen Gesellschaft für Politikwissenschaft (DGfP), Bd. 27], 167-180.

14 Dazu grundsätzlich Habermas, J.: Merkels von Demoskopie geleiteter Opportunismus, in: < www.sueddeutsche.de > vom 7. April 2011; der Gastbeitrag ist zeitgleich in der Druckausgabe unter dem Titel „Ein Pakt für oder gegen Europa? An Gründen für eine Gemeinschaft fehlt es nicht, wohl aber an einem politischen Willen - und an Verantwortung“ erschienen (Süddeutsche Zeitung vom 7. April 2011, 11).

15 Die jahrzehntelangen Schwächen der innerbayerischen Konkurrenz treten hinzu, sind aber in Teilen auch in der Sonderstellung der CSU begründet. 
tig geschaffene historische Existenzbedingung der CSU. Ihr Doppelcharakter war bereits Realität, bevor es den Nachkriegs(teil)staat überhaupt gab: Zunächst waren die Zonen und Länder das Aktionsfeld der Parteien, Bayern zugleich das Territorium mit der ausgeprägtesten politisch-kulturellen Identität. Im Gründungsprozess 1945-46 hatte sich jedoch nicht der separatistisch-konfessionelle, sondern der bundes- und interkonfessionell orientierte Flügel durchgesetzt. Die Partei operierte schon in den Übergangsinstitutionen in Arbeits- und Fraktionsgemeinschaften mit der CDU, deren bundespolitischer Gründungsakt erst 1950 vollzogen wurde. Notwendigkeiten oder Anreize für einen „reichsweiten“ $\mathrm{Zu}$ sammenschluss mit der CDU bestanden nicht. Die Langzeitwirkungen für das später entstehende westdeutsche Parteiensystem konnten nicht vorhergesehen werden; die Preisgabe der organisatorischen Unabhängigkeit stand schon seit 1948 nicht mehr zu Debatte.

Diese Selbständigkeit gilt bis heute fort. Überlegungen zur Statusänderung erstreckten sich zu keinem Zeitpunkt auf ihre Reduzierung, sondern eher auf ihre Verschärfung, sogar bis zur Herauslösung aus dem Verbund mit der CDU. Sie erwuchsen partiell aus unterschiedlichen Lagebeurteilungen und strategischen Optionen. Nach einem Vorspiel zu Beginn der 1970er Jahre verdichteten sie sich im Kreuther Beschluss von 1976, der jedoch - zumal in seinen Konsequenzen nicht gründlich durchdacht - keinen Bestand hatte. ${ }^{16}$ Im Ergebnis wurde die bayerische Position innerhalb des parlamentarischen Verbunds per Vereinbarung gestärkt. Auch die vorübergehenden Überlegungen, im deutschen Vereinigungsprozess Partner oder Standbeine in den neuen Bundesländern zu gewinnen, ${ }^{17}$ zielten auf bundespolitischen Einfluss ab, der durch die Vergrößerung des Staatsgebiets unter rein numerischen Gesichtspunkten zwangsläufig relativiert wurde. Das größte Risiko läge freilich in der Relativierung der innerbayerischen hegemonialen Position durch Konkurrenz aus dem eigenen Lager. Insofern hätte sich eine regional begrenzte Ausdehnung nur ohne den Willen oder die Fähigkeit der CDU zum Gegenschlag als sinnvoll erwiesen. ${ }^{18}$ Gleichwohl bleibt das handicap, nicht über die Option zu verfügen, den Unionsverbund aufzukündigen. Die CSU ist zwar organisatorisch selbständig, politisch aber nicht völlig frei.

16 Dazu Mintzel, A.: Geschichte der CSU. Ein Überblick, Opladen, 1977, 406-413.

17 Zum strategisch wenig durchdachten DSU-Intermezzo vgl. Müller, K.: Schwierige Machtverhältnisse. Die CSU nach Strauß, Wiesbaden, 2004, 104-116.

18 Mit Blick auf Kreuth vgl. Maier, H.: Böse Jahre, gute Jahre. Ein Leben 1931ff., München, 2011, $253 \mathrm{f}$. Vor allem: Kohl, H.: Erinnerungen 1930-1982, München, 2004, 414-438. Kohl hatte dafür gesorgt, dass die Bereitschaft der CDU zum „Einmarsch“ in Bayern in München nicht unbekannt blieb. 
Tatsächlich profitieren beide Unionsparteien von der überkommenen Konstellation. Von 1957 bis 2002 entschied die CSU die Bundestagswahlen in Bayern stets mit absoluter Mehrheit für sich, abgesehen vom Einbruch 1998 (47,7 Prozent). 2005 und 2009 entrückte zwar die absolute Mehrheit. Aber angesichts des seit 1998 dauerhaften Absinkens der CDU in ihrem Wahlgebiet unter die 30Prozent-Grenze steigt die machtpolitische Bedeutung der immer noch überproportionalen CSU-Ergebnisse sogar an. Sie stabilisieren die Position der Gesamtunion bis hin zur Grundlegung ihrer Regierungsfähigkeit. Jenseits der beiden Großen Koalitionen hat die CSU nur ein einziges Mal die Erfahrung machen müssen, zur Bildung einer unionsgeführten Koalition nicht gebraucht zu werden: in der Ausnahmesituation der ersten gesamtdeutschen Wahl 1990. Zwar hat die Wiedervereinigung das numerische Gewicht der CSU im Bund von ehedem um die 10 Prozent auf etwa 7 Prozent reduziert, politisch aber bei der Union im Kern wenig verändert. Sollte sich der Trend der Landtagswahl in Baden-Württemberg auch in den Bundestagswahlen fortsetzen, käme der bayerischen Sondersituation eine noch größere numerische Bedeutung für den Machtanspruch im Bund zu.

Ihre Doppelrolle prägt Auftreten und Agieren der CSU. Sie muss sich ihren Wählern als Sachwalterin bayerischer Interessen präsentieren, ohne den Anspruch auf gesamtdeutsche, längst auch, so vage sie sein mag, europäische Verantwortung und Gestaltung preiszugeben. Folglich genügt der Rekurs auf bayerische Interessen nicht, um ihren Forderungen in Berlin und Brüssel Nachdruck zu verleihen. Gleichwohl muss die Partei im Ergebnis dem Motto „Bayern zuerst“ huldigen, denn ihrer Dominanz dort verdankt sie ihre Stellung im Bund. Der immer wieder erhobene Anspruch, die Unionslinie bis zu begrenzten Konflikten nachhaltig mitzugestalten, ist also nicht nur Ausdruck eigenständiger Programmatik, sondern auch des existentiellen Interesses, nicht zum 16. CDU-Landesverband herabzusinken - wobei vom Erfolg dieser Doppelstrategie nicht zuletzt auch die Schwesterpartei profitiert, bei der diese Erkenntnis in letzter Zeit offenbar ein wenig in Vergessenheit geriet.

Dabei ergeben sich deutliche Wechselwirkungen zwischen Bundes- und Landesebene: In Regierungskonstellationen kann die CSU vom Aktionsbonus und ggf. vom Erfolg der von ihr mitgetragenen Bundesregierungen profitieren. Von einem bundespolitischen Malus bleibt sie aber ebenso wenig verschont. Grenzen ihrer konkurrierenden Eigenständigkeit werden in der Regel deutlich, wenn Landesund Bundesinteressen in Kollision geraten und CSU-Exponenten als wichtige Rollenträger in der Bundesregierung agieren. Beispiele sind die Divergenzen Waigels und Stoibers um die Einführung des Euro, Seehofers und zu Guttenbergs 
im Fall Opel, Söders und Aigners in der Frage der agrarischen Gentechnik. Andererseits fiel der CSU bei depressiven Krisen der größeren Schwesterpartei im Unionsverbund die Rolle der Wortführerin zu, etwa als die CDU durch den Misserfolg von 1998, als sie in ihrem Wahlgebiet erstmals seit 1949 nicht einmal 30 Prozent erreichte, sowie durch ihre Spendenaffäre tief erschüttert war und die Fortführung der Oppositionsarbeit weitgehend von bayerischen Kompetenzen und Initiativen abhängig war. In Folge dieser Situation, in der der bayerische Ministerpräsident zeitweilig als eigentlicher Oppositionsführer im Bund galt, fiel Edmund Stoiber dann auch 2002 die Kanzlerkandidatur zu.

Doppelrollen haben es an sich, situationsbedingt und -angemessen unterschiedlich interpretiert werden zu können. Auch in der Vergangenheit galt, dass der bundespolitische Mitsteuerungsanspruch aus München bei schwächerer Führungskraft der Schwesterpartei stärker hervortrat. Umgekehrt fand sich die CSU als kleinster Partner in der zweiten Großen Koalition zwischen einer präsidialen Kanzlerin und der SPD in einer deutlich schwierigeren Situation als zu Zeiten schwarz-gelber Bündnisse. Dieses Dilemma wurde durch eine Reihe interner Krisen maßgeblich und langfristig verstärkt, die das Handeln der Partei bis heute überschatten. Positionsbehauptung setzt jedenfalls intakte Parteistrukturen, überzeugendes Führungspersonal und konzeptionelle Kompetenz voraus.

\section{Machtzentren, Führung, Personalisierung}

Für die Entwicklung zur „Massen- und Apparat-Partei modernen Typs“19 erwies sich die CSU einst als bahnbrechend. Nach wie vor liegt ihre Kraft nicht zuletzt in der Parteiorganisation. Die Landesleitung, bei der organisatorische, kommunikative und partiell auch konzeptionelle Fäden zusammenlaufen, ${ }^{20}$ gilt als eines von zumindest vier Machtzentren der Partei, neben der Landtagsfraktion, der Staatsregierung und der Landesgruppe im Bundestag. Letztere erscheint nur auf den ersten Blick eigentümlich, nimmt aber grundsätzlich jene Rolle ein, welche in anderen Parteien die Fraktionen spielen. In der betont föderalen Struktur der CSU repräsentiert sie zudem die bundespolitische Dimension und ist deshalb in der Lage, ein argumentatives Gegengewicht zur Münchner Zentrale zu bilden.

19 Mintzel, A.: Anatomie, a.a.O., 67.

20 Einzelheiten: Kießling, A.: Die CSU. Machterhalt und Machterneuerung, Wiesbaden, 2004; Hopp, G.: Herzstück der CSU? Die Landesleitung zwischen Serviceagentur, Denkfabrik, und Motor der Parteimodernisierung, in: ders./Sebaldt, M./Zeitler, B. (Hg.): Die CSU. Strukturwandel, Modernisierung und Herausforderungen einer Volkspartie, Wiesbaden, 2010, 351-374; vgl. auch Müller, K., a.a.O. 
Prinzipiell hängt die Bewertung dieser Zentren ganz erheblich von personellen Faktoren und Konstellationen ab, welche durch die institutionelle Betrachtungsweise eher verdunkelt werden.

Starke Führungspersönlichkeiten als Parteivorsitzende vermögen durchaus das institutionelle Geflecht zu penetrieren und die gesamte Partei maßgeblich auf sich zu konzentrieren, zumindest zeitweise. Das gilt insbesondere dann, wenn die Ämter des Parteivorsitzenden und Ministerpräsidenten in einer Hand vereinigt sind und zusätzlich auch Bundeskompetenz vorliegt. Aber die Machtstruktur verändert sich sogleich bei Doppelspitzen, also der Trennung der Spitzenämter in Partei und Regierung, oder auch bei einer selbstbewussten, die Mitverantwortung der Fraktion in die Waagschale werfenden Fraktions- oder Landesgruppenführung. Ebenso durchdringen personelle Spezifika die Strukturen der Parteiendemokratie, wie etwa die Charakterisierung Alfons Goppels als Landesvater und seines Nachfolgers Franz Josef Strau $\beta$ als Bayernherrscher. ${ }^{21}$ Der CSU ist sogar für einige Zeit die abenteuerliche Kombination eines Ministeramtes mit dem Fraktionsvorsitz eingefallen, die sowohl für die Position des Ministerpräsidenten als auch für den Mitgestaltungsanspruch der Fraktion nicht ohne Folgen bleiben kann. Alle denkbaren Varianten (und sogar die eine, vermeintlich undenkbare) hat die Partei durchgespielt und dabei zumeist - selbst unter, gelegentlich auch gegen Strau $\beta$ und Stoiber - zu Konstellationen von Macht und Gegenmacht gefunden. Nicht nur gegen schwache (Streibl), sondern auch gegen starke und kompetente Führungsfiguren (Strau $\beta$, Waigel, Stoiber) gab es innerparteiliche Opposition und kam es zu Amtsablösungen. Gegenmacht auf Bundesebene im Unionsverbund oder auch im Bundesrat vermochten keineswegs nur Strau $\beta$ und Stoiber auszuüben, auch wenn deren Konfrontationen mit Kohl und Merkel die prominentesten Beispiele sind. Im Übrigen ist die Performance sämtlicher bayerischer Machtzentren sogar durchaus von einem bzw. einer durchsetzungsstarken und populären Unionskanzler bzw. -kanzlerin beeinflussbar.

Die aktuelle Lage ist nicht zuletzt durch den Einfluss aus Berlin gekennzeichnet, der in der Weigerung Merkels kulminierte, dem bayerischen Parteivorsitzenden Stoiber eine Gegenmachtposition am Kabinettstisch zuzugestehen. Diese Entwicklung war mitursächlich für die anschließende Führungskrise der CSU, die sie nicht nur Personal (Stoiber, Beckstein, Huber), sondern auch Vertrauen, Kompetenzzuschreibungen und Stimmen kostete. Horst Seehofer hat dann Parteivorsitz und Ministerpräsidentenamt wieder zusammengeführt, zudem auch die

21 Maier, H., a.a.O., 241-264. 
Fraktion diszipliniert, deren Vorsitzender kein Gegenpol zu sein vermag. Unter dem Stichwort „Verjüngung“ wurde das Kabinett und durch ein revirement auf der Ebene der Amtschefs der Ministerien die Verwaltung von Vertretern der Stoiber-Ära befreit oder deren Einfluss erheblich beschnitten. An den wichtigen Schaltstellen sitzt niemand mehr, der seine Position nicht der neuen Macht zu verdanken hätte, die rigide führt. Lediglich die Landesgruppe neigt bisweilen zur Eigenständigkeit. Allerdings gewann die CSU unter Seehofers Führung in Berlin unmittelbar Konfliktfähigkeit zurück, auch gegen die Kanzlerin. Seehofer profitiert innerparteilich nach der Demontage zu Guttenbergs von der Abwesenheit jeder personellen Alternative. Dessen charismatischer Aufstieg als Herausforderer offenbarte allerdings die in der Partei lange fortbestehenden Vorbehalte gegen den Vorsitzenden, die nun leer laufen. Wo in der Partei das Machtzentrum liegt, war höchstens unter Strau $\beta$ so eindeutig definiert wie derzeit ersichtlich.

Zur innerparteilichen und administrativen Frontbegradigung werden in naher Zukunft auch Positionsgewinne im Unionsgefüge sichtbar werden, die aus der aktuellen Schwäche der CDU in Baden-Württemberg resultieren. Diese hatte im Unionsverbund bisher - mit den Bayern - nicht nur für konservative Positionen, sondern auch für stabilisierende Wahlergebnisse gesorgt. Ein Resultat ihres Absturzes wird die Reduktion des Südens auf Bayern und mithin Seehofer sein. Gefährden kann ihn zum einen nur ein überzogener, quasi-autoritärer Führungsstil, der - siehe Stoiber - zwangsläufig Widerstand provoziert, zum anderen das Absinken der CSU unter die 40-Prozent-Marke. Seehofers zum Populismus neigende politische Führungslinie ist allerdings von dem Bestreben bestimmt, 2013 im Landtag die absolute Mehrheit der Mandate zurückzugewinnen, was sicher nicht nur ihm wichtiger ist als die Perfektionierung innerparteilicher, innergouvernementaler oder innerfraktioneller Mitbestimmung. Die sich abzeichnende Stärkung seiner bundespolitischen Rolle, die zudem eine persönliche Genugtuung angesichts früherer Konflikte mit Angela Merkel darstellt, könnte dieses Unterfangen stützen. Worin aber bestehen die substantiellen bayerischen Beiträge zur Bundespolitik?

Diese Frage stellt sich nicht unabhängig von der Entwicklung der Parteien und des Parlamentarismus, die wiederum graduell mit jenen gesellschaftlichen Veränderungen korrespondiert, die eingangs bereits angedeutet wurden. Eine entpolitisierte Gesellschaft zeigt am partizipatorischen politischen Diskurs wenig Interesse. Die Mediendemokratie orientiert sich am event, nicht an der Substanz. Zur Bewältigung komplexer Probleme und zur raschen Reaktion gezwungene Führungen meinen, Entscheidungsspielräume stets in Anspruch nehmen zu müs- 
sen: Innerparteiliche und innerparlamentarische Legitimationsprozeduren werden dadurch (nicht nur bei supranationalen Entscheidungen!) ausgehöhlt. Die Charakterisierung des gegenwärtigen Zustands als Postdemokratie und Postparlamentarismus $^{22}$ mag übertrieben sein, zeigt aber unzweifelhaft reale Tendenzen auf.

Wenn sich politische Prozesse, politische Institutionen und politische Öffentlichkeit derart wandeln, könnte die (im Kern nicht nur für Bayern) skizzierte Personalisierung politischer Führung sowie ein themen- und entscheidungsorientierter Populismus eine instinktsichere Reaktion darstellen und einen Zukunftstypus repräsentieren, der sich mit normativen Vorgaben erheblich weniger deckt als mit aktuellen Interessenlagen. Franz Josef Strauß Maximen, es komme nicht darauf an, wie etwas ankomme, sondern was getan werden müsse - und: man solle dem Volk aufs Maul schauen, ihm aber nicht nach dem Munde reden liefen in einer medien- und emotionsgesteuerten Stimmungsdemokratie ${ }^{23}$ leer. Kontinuität, Rationalität und Grundsatztreue besäßen bestenfalls eingeschränkte Relevanz. Die aktuelle von Bayern forcierte atompolitische Wende trägt prägnante Züge von all dem.

Konzeptionelle Politikansätze wie unter Stoiber, Beharrung auf übergreifenden Grundsatzoptionen wie unter $\operatorname{Strau} \beta^{24}$ oder alles andere als opportunistische Verteidigungsstrategien zugunsten ethischer Orientierungen ${ }^{25}$ wie unter Waigel passen nicht mehr in ein derart gewandeltes Politikverständnis, dem sich die Partei und ihre Führung nicht wirklich entziehen können. Für einen gewissen heroischen Konservatismus ist sie selbst nicht mehr ausreichend homogen. Da-

22 Hierzu statt vieler prominent Crouch, C.: Postdemokratie, Frankfurt a. M., 2008; Andersen, S.S./Burns, T.R.: The European Union and the Erosion of Parliamentary Democracy. A Study of Post-Parliamentary Governance, in: Andersen, S.S./Eliassen, K.A. (Hg.): The European Union: How Democratic Is It?, Beverly Hills/London, 1996, 227-251; darüber hinaus Benz, A.: Postparlamentarische Demokratie? Demokratische Legitimation im kooperativen Staat, in: Greven, M. (Hg.): Demokratie - eine Kultur des Westens? 20. Wissenschaftlicher Kongress der Deutschen Vereinigung für Politische Wissenschaft, Opladen, 1998, 201-222; Jörke, D.: Auf dem Weg in die Postdemokratie, in: Leviathan, 33/4 (2005), 482-491; Helms, L.: Ist die Bundesrepublik eine „Post-Demokratie“? Eine Analyse am Schnittpunkt von Demokratieforschung und Vergleichender Regierungslehre, in: ZSE 8/2 (2010), 202-227; Mouffe, Ch.: „Postdemokratie“ und die zunehmende Entpolitisierung, in: Aus Politik und Zeitgeschichte, 61/1-2 (2011), 3-5.

$23 \mathrm{Zu}$ den ersten Beobachtungen bereits Oberreuter, H.: Stimmungsdemokratie. Strömungen im politischen Bewußtsein, Zürich/Osnabrück, 1987.

24 Als ein derartiges Beispiel sei der Gang nach Karlsruhe gegen den Grundlagenvertrag zwischen BRD und DDR genannt, der wichtige, im späteren Vereinigungsprozess hilfreiche Grenzen zog.

25 Prominentes Beispiel: Anrufung des Bundesverfassungsgerichts zu §218 StGB. 
mit normalisiert sich zugleich ihre Fähigkeit zum Agenda-Setting und zu Konflikten - aber sie entschwindet nicht.

Personalisierung der Führung und Entdramatisierung des Politischen müssen zwangsläufig Institutionen transzendieren. Sicher ist die Landesgruppe nach wie vor das wichtigste Instrument zur kontinuierlichen Umsetzung des bundespolitischen Mitgestaltungsanspruchs und ihr Vorsitz ein anerkanntes Führungs- und Aufstiegsamt. Sie ist routinemäßig „Transmissionsriemen“26 zwischen München und dem Bund und ihre ,strategische Schlüsselstellung““27 als Fraktion in der Fraktion mit eigenen Rechten, eigener Infrastruktur und eigener Konkurrenzfähigkeit im Fraktionsverbund besteht grundsätzlich fort. Unzweifelhaft ist sie in der Partei das am wenigsten mediatisierte Machtzentrum. Als parlamentarische Gruppierung partizipiert sie aber grundsätzlich an den geschilderten Entparlamentarisierungstendenzen und als Institution unterliegt sie Entmächtigungen durch die impliziten Folgen personalisierter Parteiführung und die dadurch politisch begrenzte Möglichkeit zum Konflikt. Wenn ihr Positionsverluste attestiert werden, ${ }^{28}$ dürfen diese durchaus strukturellen Gründe sowie wechselnde Rahmenbedingungen nicht außer Acht gelassen werden. So ist die Landesgruppe intern sicher am mächtigsten, wenn die Union in der Opposition agiert und der Parteivorsitzende ihr angehört. Aber gestalten kann sie in dieser Konstellation nicht. Dies wiederum gelingt, wenn die Union regiert und der Parteivorsitzende in München agiert. Allerdings verliert die Landesgruppe dann als Machtzentrum in der Partei an Bedeutung.

\section{Programmatische Grundlagen}

Wenn Parteien die sich wandelnden und modernisierenden gesellschaftlichen Rahmenbedingungen in Aktions-, Kommunikations- und Führungsformen aufgreifen, muss sich das auch auf ihre programmatische Orientierung auswirken. Wie steht es dabei um die Integrationsfähigkeit der Parteiprogramme?

26 Waigel, T.: Die Rolle der CSU-Landesgruppe im Spannungsfeld von Partei, Regierung, Fraktion, in: Politische Studien, Sonderheft 1 (1989), 106-112 (111).

27 Mintzel, A.: Die Rolle der CSU-Landesgruppe im politischen Kräftespiel der Bundesrepublik Deutschland, ebd., 113-134 (123).

28 Müller, K., a.a.O., 95-104 schon für die 80er Jahre. Differenzierter Kießling, A., a.a.O., 134-146; deutliche Hinweise auf den Einfluss wechselnder Rahmenbedingungen bei Hempel, Y.: Statthalter der Partei oder Juniorpartner der Union? Die CSU-Landesgruppe in Berlin, in: Hopp, G./Sebaldt, M./Zeitler, B. (Hg.), a.a.O., 287-308. 
Die CSU hat sich seit 1946 sechs Grundsatzprogramme gegeben, die sich vom traditionalistischen Verständnis einer Weltanschauungspartei über pragmatischere Definitionen christlicher Politik bis hin zur Wiederentdeckung zeitgemäß interpretierter normativer Orientierungen entwickelten. Die Kohärenz dieser Programme erwächst aus der Priorität wertorientierter politischer Steuerung ohne ideologische Verengung. Wenn sogar die traditionell eigenen sozialmoralischen Milieus sich in den Individualisierungs- und Pluralisierungsprozessen der Gegenwart verlieren, so verschwinden erst recht die festen Zielgruppen programmatischer Selbstvergewisserung und Selbstgewissheit. Schon Mitte der 1970er Jahre kam Franz Josef Strau $\beta$, ausgehend vom höchsten Wert der Freiheit, die als Kern des Christlichen definiert war, zu dem Schluss, Parteien könnten keine Wertordnung als bindend auferlegen. Das Individuum müsse frei entscheiden, und die Partei könne nur die Vorkehrungen schaffen, die es ihm ermöglichten, eine echte Wertewahl zu treffen. Programmierbar sei nur Offenheit. ${ }^{29} 2007$ ist im Grundsatzprogramm nicht mehr christliches Bekenntnis das höchste Gebot, sondern „nur“ die Anerkennung seiner Werte, wodurch die Partei sich dezidiert auch für Nichtchristen und grundsätzlich gesellschaftlich weiter öffnet. So tritt z.B. neben Ehe und Familie der Respekt für alle Lebensgemeinschaften, in denen Partner verlässlich Verantwortung füreinander tragen. Das „C“ wurde zunehmend in seine individuellen und gesellschaftlichen Bezüge übersetzt und keineswegs exklusiv in Anspruch genommen, zugleich aber stets auf die Würde der Person bezogen - nicht zuletzt angesichts der ethischen Herausforderungen des wissenschaftlichen Fortschritts, etwa in der Gen- und Biotechnologie.

Orientierung an der Menschenwürde, Offenheit, ökonomische und gesellschaftliche Modernisierung - ist das konservativ? Erst im Grundsatzprogramm von 1968 hat sich die CSU dazu bekannt, ,,auch“ eine konservative Partei zu sein. 2007 steht dann der Bezug zum Konservativen neben sieben anderen Orientierungen wie etwa christliche, soziale, europäische und internationale Verantwortung, Freiheit sowie Bewahrung der Schöpfung. Darauf lässt sich keine prinzipielle Gegenposition zur Moderne stützen. Ihr konservatives Element wird von der Partei selbst unter der Überschrift „Moderne wertorientierte Volkspartei“ präsentiert. ${ }^{30}$ Als politischer Begriff ist Konservatismus höchst ambivalent und wurde

29 Strauß, F.J.: Das Verhältnis von Programm und Pragmatismus in der politischen Praxis, in: Die Grundsatzdiskussion in der CSU, hg. v. P. Gutjahr-Löser und T. Waigel, München, 1977, 19.

30 Dabei gründet ,[i]hre Politik [...] auf der dauerhaften Wertordnung des abendländischen Denkens sowie dem geschichtlichen und kulturellen Erbe unseres Volkes. Tradition und Heimat, Sprache und Kultur geben den Menschen Geborgenheit und der Gemeinschaft Zusammenhalt. Die CSU misst das Neue am 
inzwischen, anders als zu Zeiten Edmund Burkes, der den Umbrüchen seiner Zeit ein eindeutiges „Anti“ entgegenrief, aussagelos - eher ein Kampfbegriff der politischen Gegner. Programmdiskussion und Praxis der Partei scheinen die These des Politikphilosophen Henning Ottmann aufzugreifen, derzufolge ein „lebensfähiger Konservativismus beides zu meiden“ habe: „unversöhnliche Gegnerschaft zur Moderne“ und ,die kritiklose Anerkennung derselben [...]. Konservativismus ist Kritik und Apologie der Moderne zugleich und er ist in seinen besten Formen Versuch, jene nicht modernen Bedingungen der Möglichkeit moderner Freiheit zu bewahren, die diese selbst nicht garantieren kann. “31

Freiheit, Offenheit und Wertordnung des abendländischen Denkens, dem die Aufklärung (und politisch die republikanische Demokratie!) untrennbar zugehören - nach all dem kann das „feste Wertefundament“ für gesellschaftliche Kooperation und Zukunftsverantwortung, das ein Papier der Hanns-Seidel-Stiftung als Merkmal konservativen Denkens apostrophiert, nur in der Autonomie des Individuums bestehen, in der Freiheit und Verantwortung zusammengedacht sind.

Das wirft in der Aktualität drei Probleme auf. Erstens: Dieses gleichwohl starke Fundament repräsentiert weder weltanschauliche Gewissheiten noch zeitgeistliche Beliebigkeiten. Es verlangt herausfordernde, offensichtlich gelegentlich auch überfordernde politisch-intellektuelle Anstrengungen in der praktischen Umsetzung. Zweitens: Angesichts der beschriebenen kurzfristig orientierten Opportunitäts-, Popularitäts- und Stimmungsabhängigkeiten der modernen Demokratie stellt sich immer deutlicher die Frage nach der grundsätzlichen Relevanz solcher Wertorientierungen, die im Vollzug instinktsicherer Alltagsreaktionen eher nachrangig erscheinen. Drittens: Selbstbestimmung in (verantworteter) Freiheit ist für viele Anhänger der C-Parteien noch immer ein ihr überkommenes Denken in festen Ordnungen herausfordernder Reizbegriff und wenigstens theoretisch ein Spaltpilz zwischen aufgeklärterer politischer Führung und weniger aufgeklärter Anhängerschaft.

Mit der Akzentuierung einer ,aktiven Bürgergesellschaft“ sowie einer „solidarischen Leistungsgesellschaft“, mit reformierten gesellschafts- und familienpoliti-

Bewährten und stellt Fortschritt in den Dienst der Menschen. Die CSU gestaltet eine langfristig orientierte, nachhaltige Politik zum Schutz des Lebens und für die Zukunft kommender Generationen“ (Grundsatzprogramm der Christlich-Sozialen Union in Bayern. Chancen für alle! In Freiheit und Verantwortung gemeinsam Zukunft gestalten, München, 2007, 180).

31 Staatslexikon, Bd. 3, 7. Aufl. 1995, 640. 
schen Aussagen, mit sozialen, ökologischen und ökonomischen Leitlinien unter dem Gesichtspunkt der Europäisierungs- und Globalisierungsprozesse verleiht das Grundsatzprogramm von 2007 der Partei „Fitness“ und Zukunftsfähigkeit soweit Programme dazu überhaupt noch einen Beitrag leisten. Es hätte vielleicht auch jene Wählergruppen ansprechen können, welche die CSU in den Jahren 2008 und 2009 nicht mehr erreichen konnte. In den Turbulenzen der Führungskrise, der Ablösung Stoibers und der Installation der Doppelspitze BecksteinHuber ging es allerdings weitgehend unter. Die Chance, den Eindruck eines von den Menschen abgehobenen, orientierungslosen innerparteilichen Machtspiels aufzusprengen, stand ihm damals nicht offen. Schon diese Entstehungssituation hat ihm Wirkung und Dynamik genommen, zumal die eher aktualitäts- und instinktorientierte Führung sich seit dem Wechsel zu Seehofer weniger programmatisch orientiert. Immerhin mutet die Spitze der Basis erhebliche, alles andere als rückwärtsorientierte Neuorientierungen zu, wie etwa die durchaus brachiale Durchsetzung der Frauenquote oder des Ausstiegs aus der Kernenergie.

Prinzipiell tritt die CSU operativ seit langem für die soziale Marktwirtschaft (unter Betonung ihrer sozialen Dimension), für die integrationspolitisch relevante Erhaltung der kulturellen Identität im Wandel $^{32}$ und für ein abgewogenes Verhältnis von Freiheit und innerer Sicherheit ein. Zudem ist sie in Deutschland der stärkste Hort des Föderalismus, von der Schöpfung des Grundgesetzes bis hin zur Schutzklausel im Fraktionsvertrag mit der CDU, in föderalen Angelegenheiten nicht überstimmt werden zu dürfen. Schließlich ist der Föderalismus conditio sine qua non für die Sonderstellung der Partei.

Sämtliche Grundorientierungen der Partei spiegeln sich in ihren europäischen Positionen wider, die von den politisch-kulturellen Gemeinsamkeiten und den auf dieser Basis ausgeprägten nationalen Identitäten ausgehen. Von daher bestimmt sie für sich die Grenzen der EU und weitere Beitrittsperspektiven nach der Zugehörigkeit zu den geschichtlichen und kulturellen Gemeinsamkeiten. Im Spannungsfeld zwischen Integration und Unifikation reagiert die CSU demgemäß höchst sensibel und unter Rekurs auf die Subsidiarität als Ordnungsprinzip der EU, wobei sie die europäische Subsidiaritätsklausel bei Bedarf auch auf der nationalstaatlichen Ebene (wo sie ohnehin präziser ausgeprägt ist) zur Geltung bringt; in Berlin unterstützend, in Brüssel defensiv, um Kompetenzeinbußen abzuwehren. An Größe und Wirtschaftskraft mit den Niederlanden vergleichbar, sehen sich die Bayern durchaus als eigenständige Mitspieler in der europäischen

32 Begleitet von Toleranz und Offenheit ist das nicht unbedingt „konservativ“. 
Arena, zumal dort föderale Strukturen eher unvertraut sind. Die regionalen Ansätze in der EU bis hin zum Ausschuss der Regionen (den man sich allerdings effizienter gewünscht hätte) sind bayerisch beeinflusst. Niemand sucht nachhaltiger nach Schranken und Kompensationen für den Machtverlust der nationalen und subnationalen Parlamente. Niemand ist sich auch der Relativität der Subsidiaritätsidee sowie des Placebo-Effekts des sogenannten Frühwarnsystems und der Subsidiaritätsrüge mit einem in den vorgesehenen Fristen nicht erreichbaren Drittel der den nationalen Parlamenten zugewiesenen Stimmen so bewusst. ${ }^{33}$

CSU und Staatsregierung setzen daher auf eigene Präsenz und Handlungsfähigkeit, wobei die kleine Gruppe der Abgeordneten nicht in ihren Wirkungsmöglichkeiten mit der Berliner Landesgruppe verglichen werden kann, aber doch eine Aktionseinheit darstellt - ohne, wie alles institutionell Europäische, in der Öffentlichkeit signifikant präsent zu sein. In Brüssel gilt aber genau dies für die Bayerische Landesvertretung im Schlösschen Pasteur inmitten der europäischen Institutionen, die unabhängig vom Bund sichtbar und selbständig bayerische Interessen zum Ausdruck bringt, ohne penibel nach Zuständigkeiten zu fragen.

Im Kern beherrscht die Interessenvertretung die Aktionsweisen von Staatsregierung und Partei - in Berlin wie in Brüssel. Zugespitzt: Nicht die Fortentwicklung der EU steht im Fokus, sondern die Effektivierung und Verteidigung eigener Ansprüche in der EU. Bayern gilt stets die primäre Aufmerksamkeit. Anderenorts ist dies nicht anders, bei der CSU aber deutlich wirksamer.

\section{Zum Zustand der Partei}

In welchem Zustand findet sich die Partei heute? Wird sie ihre Sonderrolle bewahren können? Indikatoren ihrer Herausforderung wurden bereits benannt: Einbrüche am Wählermarkt, Verlust der Hegemonie und Zwang zur (noch immer mental nicht angenommenen) Koalitionsbildung, ambivalente Durchsetzungschancen im Bund, Wandel des Politikverständnisses bei Parteien, Wählern und Medien, Auswirkungen auf das Führungsverhalten. Darauf sind nicht nur Einflussschwankungen zwischen den Machtzentren zurückzuführen. Soweit sich die Gestaltungs- und Akzeptanzchancen der Institution ,politische Partei“ in der Gesellschaft grundlegend, und zwar negativ, verändern, gehen diese Prozesse auch an der CSU nicht vorbei, die entsprechenden Analysen allerdings nur un-

33 Dazu die nüchterne Analyse von Bocklet, R. in: 40 Jahre Deutsche Vereinigung für Parlamentsfragen, Baden-Baden, 2011, 97-105. 
gern zuhört und verbal den Eindruck erweckt, mit traditionellen Rezepten die Moderne zwingen zu können, während sie programmatisch mit eben diesen Traditionen bricht. Für die ständig schwindenden Stammwähler zu reden und zeitgleich für modernere Segmente der Gesellschaft praktisch zu handeln, führt zu Fragen nach Konsistenz, Klarheit und Glaubwürdigkeit.

Zwar partizipiert die CSU an den Erosionsprozessen der Volksparteien, doch steht sie in Relation noch immer besser da. Zwischen 1991 und 2011 erlitt auch sie einen eindeutigen Mitgliederschwund (-17 Prozent), wenngleich nicht in ähnlichem Ausmaß wie die CDU (-26 Prozent) und die SPD (-46 Prozent). ${ }^{34}$ Die Daten spiegeln den regionalen Vorteil, nicht zwischen Flensburg und Freiburg, Düsseldorf und Dresden integrieren zu müssen, sondern nur zwischen Hof und Garmisch-Partenkirchen. Die Beschleunigung des Rückgangs in den letzten anderthalb Jahren (-6.900, d.h. -4 Prozent) zeigt aber, dass die Vorteile der regionalen Repräsentation die Partei nicht dauerhaft schützen werden, auch wenn sich die Folgen der Krisenjahre seit 2007 noch in den Verlusten spiegeln. Zugleich ist die Mitgliedschaft deutlich überaltert.

Gefahrenpotentiale lauern insbesondere in Aufweichungstendenzen der ehedem traumhaften Verankerung im ,,vorpolitischen Raum“, wofür etwa die feindselige Interessendifferenzierung in der Landwirtschaft signifikant ist, die den Bayerischen Bauernverbund und dessen Symbiose mit der CSU erschütterte und offenbar zur überproportionalen Abwanderungsbewegung der Landwirte bei der letzten Landtagswahl geführt hat. Auch sind jüngere Mandatsträger nicht mehr in gleicher Weise in Vereinen und Verbänden engagiert, die das politische und gesellschaftliche Kommunikationsfeld im Nahraum dominieren.

Nach wie vor ist die Organisationsstruktur konkurrenzlos und flächendeckend intakt - oberflächlich gesehen. An der politischen Aktivität vieler Ortsverbände erheben Experten Zweifel, speziell was die Erörterung politischer Themen jenseits des Lokalen angeht. Insofern ist die Partei zwar da, aber vielfach nicht präsent. Zusätzlich dürfte bei einem Durchschnittsalter der Mitglieder jenseits des sechzigsten Lebensjahres, von denen rund 80 Prozent nicht wirklich per E-Mail erreichbar sind, eine internetbasierte „Mitmach-Offensive“ zur Förderung innerparteilicher Willensbildung wenig zielführend sein.

34 Im Gegensatz zu dieser negativen Entwicklung der Volksparteien verbuchten die Grünen im selben Zeitraum einen Mitgliederzuwachs von insgesamt 46 Prozent - freilich ausgehend von einem niedrigeren Niveau (eigene Berechnungen auf Grundlage von Angaben der Parteien). 
Peter Glotz hat vor Jahrzehnten die großen Parteien als schwer bewegliche Tanker beschrieben. ${ }^{35}$ In Abständen wiederkehrende Erörterungen über die Notwendigkeit von Reformen bestätigen diese Beobachtung. Denn sie behandeln seit langem ohne reale Konsequenzen die gleichen Themen. Die CSU hat unter dem Stichwort „Mitmachpartei“ im Juli 2009 ein „Leitbild 2010 plus“ erstellt und auf dem Parteitag Ende Oktober 2010 verabschiedet, das von vermehrten Partizipationsbegehren von Bürgern und Mitgliedern ausgeht und offene Diskussionen auf allen Ebenen - auch mit Nichtmitgliedern - einfordert, bis hin zur Mitgliederbefragung. Allerdings besteht nicht der Eindruck, dass bei wirklich wichtigen Entscheidungen an deren Einsatz gedacht wird, was etwa bei den Turbulenzen um den Ausstieg aus der Kernenergie ebenso interessant wie kontraproduktiv gewesen wäre. Wichtiges wird sich keine Führung je aus der Hand nehmen lassen. Welche Substanz jenseits des Placebos und Modernitätsanstrichs kommt solchen Instrumenten dann aber zu?

Im April 2011 startete ein Bundestagsabgeordneter eine Mitgliederbefragung ausgerechnet zur Präimplantationsdiagnostik, verbunden mit einer Kurzübersicht über die drei dem Bundestag vorliegenden Anträge und dem Hinweis, sich eigene Gedanken gemacht zu haben: „Das Umfrageergebnis stellt für mich aber eine Richtschnur dar und gibt mir zudem Orientierung hinsichtlich des Meinungsbildes unserer Mitglieder vor Ort. ${ }^{\text {36 }}$ Könnte ein Abgeordneter sein Mandat verantwortlich wahrnehmen, übergäbe er (was der Wortlaut seines Schreibens nicht nahelegt) eine der wenigen Gewissensentscheidungen eines Parlamentariers an die Parteimitglieder? Andererseits: Worin läge deren Mitmachpotential, wenn sie ihrem Abgeordneten in einem quasi förmlichen Verfahren ihre wie auch immer reflektierte Meinung deutlich, aber wohl folgenlos kundtun können?

Interessantere Partizipationschancen fänden sich etwa in der Urwahl von Kandidaten für Parlamentsämter durch die Mitglieder sowie mit Blick auf unmittelbaren Einfluss auf die Reihung der Listenkandidaturen. Daran besteht allerdings kein Interesse der Parteieliten. Immerhin wurde die Belebung innerparteilicher Demokratie als Thema erkannt.

35 Glotz, P.: Die Beweglichkeit des Tankers, München, 1982.

36 Schreiben von Andreas Scheuer, MdB an die CSU-Mitglieder von Passau Stadt und Land vom 15. April 2011. 


\section{Fazit: offene Zukunft der CSU}

Ein knappes Fazit führt zu drei wesentlichen Erkenntnissen:

- Nach den Turbulenzen um Stoibers Sturz zeichnen sich durchaus positive Trends ab, die personelle Erneuerung scheint heute vollzogen. Sie hat sich nach zu Guttenbergs Fall (der die Partei eines nicht leicht zu ersetzenden auBenpolitischen und transatlantischen Experten beraubte) auch dadurch gefestigt, dass keine unmittelbare Führungsreserve mehr vorhanden ist. Wenn Vorbehalte gegenstandslos und konkurrierende Machtzentren im Wesentlichen mediatisiert werden, dient dies der internen Stabilisierung. Zugleich fehlt es der Partei ausweislich ihres neuen Grundsatzprogramms nicht an konzeptionellen Auspizien, bei denen sie durch die Etablierung einer ,Zukunftskommission“ auch auf der Höhe der Zeit zu bleiben gedenkt. Präsenz und Konfliktfähigkeit auf Bundesebene wurden zurückgewonnen, durch die Schwäche der baden-württembergischen Union sogar mit verstärkten Aktions- und Wahrnehmungschancen.

- Gleichwohl sind Einbußen der Sonderstellung in Bayern und im Bund zu konstatieren, die sich aus massiven Einbrüchen am bayerischen Wählermarkt, den damit verbundenen Vertrauens- und Identitätsverlusten sowie dem Entschwinden der Hegemonie in Bayern ergeben, aber auch aus einer erstaunlichen Wiederkehr der Kanzler(innen)demokratie in Berlin, die von ihren Amts- und Machtressourcen rigiden Gebrauch macht, auch gegenüber der kleineren Schwesterpartei, auf die sie eigentlich zum Machterhalt angewiesen ist. Dieser steht allerdings kein geeignetes „Erpressungspotential“ zur Verfügung. Gleichwohl könnten sich Kanzler(innen)demokratie und Koalitionsnotwendigkeiten als nur vorübergehende Erscheinungen erweisen. Inbesondere mit Blick auf die Notwendigkeit politischer Bündnisse erscheint dies allerdings eher unrealistisch.

- Bleiben werden die grundlegenden Herausforderungen, denen der Typus der Volkspartei aufgrund der gesellschaftlichen Individualisierungs-, Pluralisierungs- und Säkularisierungsprozesse unterliegt. Sie untergraben die Potentiale der größeren und fördern offensichtlich die der mittleren und kleineren Parteien, hinterlassen jedoch immer weniger Berechenbarkeit und Verlässlichkeit aufgrund der Volatilität der Wählerschaft. Nicht zuletzt werden daraus neue Koalitionsarithmetiken entstehen, auch mit Schwarz-Grün als relevanter Option. Dadurch wird die CSU wie bisher schon unter Wandlungsdruck stehen und sich zunehmend - wie die CDU seit langem - mit der Frage nach Wertorientierung und Markenkern konfrontiert sehen. 